\title{
Broadband RCS Reduction by a Quaternionic Metasurface
}

\author{
Zhao Zhang ${ }^{1}$, Yazhong Zhang ${ }^{2}$, Tianlong $\mathrm{Wu}^{2} \mathbb{}{ }^{\mathbb{D}}$, Shaowen Chen ${ }^{2}$, Wei $\mathrm{Li}^{2, *}$ and Jianguo Guan ${ }^{2} \mathbb{D}$ \\ 1 The First Aircraft Institute of AVIC, Xi'an 710089, China; hylonzz@sina.com \\ 2 State Key Laboratory of Advanced Technology for Materials Synthesis and Processing, \\ International School of Materials Science and Engineering, Wuhan University of Technology, \\ Wuhan 430070, China; yuezheng@whut.edu.cn (Y.Z.); tlwuoinc@whut.edu.cn (T.W.); \\ chen_shaowen@whut.edu.cn (S.C.); guanjg@whut.edu.cn (J.G.) \\ * Correspondence: wellee@whut.edu.cn
}

check for

updates

Citation: Zhang, Z.; Zhang, Y.; Wu,

T.; Chen, S.; Li, W.; Guan, J.

Broadband RCS Reduction by a Quaternionic Metasurface. Materials 2021, 14, 2787. https://doi.org/

$10.3390 /$ ma14112787

Academic Editor: Maria Principe

Received: 22 April 2021

Accepted: 18 May 2021

Published: 24 May 2021

Publisher's Note: MDPI stays neutral with regard to jurisdictional claims in published maps and institutional affiliations.

Copyright: (c) 2021 by the authors. Licensee MDPI, Basel, Switzerland. This article is an open access article distributed under the terms and conditions of the Creative Commons Attribution (CC BY) license (https:/ / creativecommons.org/licenses/by/ $4.0 /)$.

\begin{abstract}
A quaternionic metasurface consisting of two pairs of units with destructive phase difference is proposed to extend the bandwidth of radar cross section (RCS) reduction. The two pairs of units are designed to have complementary phase-different bandwidth, which extends the bandwidth of RCS reduction. The overlaps of their bandwidth enhance the RCS reduction, resulting in a metasurface having broadband and strong RCS reduction. This design and the wideband RCS reduction of the quaternionic metasurface were verified by analytical calculation with superposition principle of electric field, numerical simulation with commercial software package CST Microwave Studio and experiment in microwave anechoic chamber. The scattering mechanism and the angular performance of the quaternionic metasurface were also investigated.
\end{abstract}

Keywords: metasurface; absorbing material; metamaterial; RCS reduction

\section{Introduction}

In recent years, metamaterials attract extensive research interests because of their brandnew electromagnetic mechanism, designable material parameters and powerful ability in controlling reflection, propagation, and absorption of electromagnetic waves [1-4]. Metasurfaces are two-dimensional metamaterials which consist of sub-wavelength unit cells arranged in a plane in a periodical or deliberately disordered manner [5], offering extra advantages of low profile when inheriting the flexible design and outstanding performance of metamaterials [6]. In view of the advantages of the metamaterials/metasurfaces and the importance of microwave absorbing materials in both military and civilian applications $[7,8]$, they are soon crossed over as metamaterial absorbers [1,9-13] or radar cross section (RCS) reducers [14,15], and become an important and prospective research field.

Metamaterial absorbers usually show narrow working bandwidth that is not favored for many practical applications [16,17]. Numerous efforts have been made to extend the bandwidth of metamaterial absorbers, typically by integrating multiple unit cells in plane or thickness didrection [18,19], usually resulted in multiple absorption peaks or even a broadband absorption bandwidth. The pyramidal metamaterials, which are constructed by stacking tens of layers of size gradient resonant unit cells, can realize an absorption bandwidth comparable to that of the best-performing $1 \mathrm{~mm}$ thick traditional magnetic absorbers in the range of 8-18 $\mathrm{GHz}$ [20-22]. Different from metamaterial absorbers which directly reduce the amplitude of microwaves by absorption, metasurfaces exploit the phase modulation of gradient changing unit cells to reduce backscattering by interference $[23,24]$. Based on this idea, the unit cells of metasurfaces with different phases are coded, designed, and programmed to show highly designable scattering patterns, including the ones that show extremely low radar observability [25-28]. The RCS reduction metasurfaces makes use of phase difference of neighboring unit cells other than the strong resonant absorption, granting them much broader working bandwidth and lower profile with respect to metamaterial absorbers [29,30]. For example, Cui et al. proposed a metasurface that composed of different square patches with a thickness of 
only $1.985 \mathrm{~mm}$ to exhibit a $-10 \mathrm{~dB}$ RCS reduction in the frequency range of 7.8-12 GHz [31]. To simplify the design and fabrication of these metasurfaces, they are usually constructed by a pair of unit cells with opposed phases to form a chessboard-like arrangement. The frequency range of effective phase difference is closely related to the thickness of the dielectric layer, the frequency band of effective phase difference is limited for a certain thickness of the dielectric layer. Therefore, it is difficult to generate effective phase difference in the wideband by only a pair of unit cells.

In this work, a quaternionic metasurface with two pairs of anti-phase unit cells is proposed to achieve broadband RCS reduction. The two pairs of unit cells are designed to have opposed reflection phase in different frequency range. Consequently, the combination of them shows extended working bandwidth with respect to existing binary schemes that involves only one pair of anti-phase unit cells. The final RCS reduction bandwidth of $-10 \mathrm{~dB}$ is from 13 to $32 \mathrm{GHz}$, which is verified by experimental measurements.

\section{Design Principle of Metasurface}

With the normal incidence of electromagnetic waves, the monostatic RCS reduction by a metasurface consisting with $\mathrm{M} \times \mathrm{N}$ units can be expressed as:

$$
R C S R_{\text {normal }}=20 \log \left|\sum_{m=1}^{M} \sum_{N=1}^{N} E_{m n} e^{i k \beta_{m n}}\right|
$$

where $E_{m n}$ and $\beta_{m n}$ represent the amplitude and phase of the reflected electric field of the unit in row $m$ and column $n$ of metasurface, respectively, and $k$ is the wave vector of the incident electromagnetic wave in vacuum. The RCS reduction of the metasurface can be calculated by substituting the reflected amplitude and phase of all units into Equation (1). From Equation (1) we can also obtain the RCS reduction materials with the versatile and controllable properties by using different units. However, multifarious types of units will also complicate the design strategy and technical process. Consequently, the types of unit cell should be as less as possible in the premise of meeting the aimed requirements. In the simplest case, the RCS reduction formula of the chessboard metasurface with only two kinds of unit cells can be significantly simplified as:

$$
R C S R=20 \log \left|\frac{A_{1} e^{i k \beta_{1}}+A_{2} e^{i k \beta_{2}}}{2}\right|
$$

where $A$ represents the amplitude of the reflected electromagnetic waves and the numeric subscripts correspond to different type of units. According to Equation (2), the RCS reduction can be achieved to some extent if the phase difference between unit ranges within $180 \pm 60^{\circ}$, and $-10 \mathrm{~dB}$ RCS reduction can be achieved when the phase difference ranges within $180 \pm 37^{\circ}$. The RCS reduction formula for the metasurface, which is composed of two pairs of units (four types of units) can be described as:

$$
\operatorname{RCSR}_{4 \text { units }}=20 \log \left|\frac{A_{1} e^{i k \beta_{+}}+A_{1} e^{i k \beta_{-}}+A_{2} e^{i k \beta_{+}}+A_{2} e^{i k \beta_{-}}}{4}\right|
$$

the subscripts 1 and 2 represent the index of the paired metamaterial units, and $\beta_{+}$and $\beta_{-}$represent metamaterial units with positive and negative phases. According to Equation (3), the RCS reduction below $-10 \mathrm{~dB}$ can be achieved as we obtain the same reflection amplitudes and the phase difference ranges within $180^{\circ} \pm 37^{\circ}$ at the same time. One can also realize a good RCS reduction and the expansion bandwidth if only one pair of phase difference meets the $180^{\circ} \pm 37^{\circ}$ condition and the other pair meets the condition of $180^{\circ}$ $\pm 60^{\circ}$. Since the working band and reflection characteristics of the two pairs of cells can be designed separately, it can be expected that the quaternionic design possesses better wideband performance compared to the existing set of binary designs. 


\section{Results and Discussion}

\subsection{Anti-Phase Unit Cells}

The structural schematics of metamaterial units with the basic shapes are square plates and square rings, labeled as $\mathrm{E}^{\#}$ and $\mathrm{E}^{\#}$, as shown in Figure $1 \mathrm{a}, \mathrm{b}$. The pitch of both structural units is $p=6 \mathrm{~mm}$, and other geometric parameters are $l p=2.8 \mathrm{~mm}, l r=4.5 \mathrm{~mm}$, and $w r=0.5 \mathrm{~mm}$. The thickness of metal structures with the conductivity of $5.8 \times 10^{7} \mathrm{~S} / \mathrm{m}$ and dielectric spacer F4B plate with the complex permittivity of $\varepsilon_{r}=2.65(1-0.001 i)$ are $t=0.035 \mathrm{~mm}$ and $d=2 \mathrm{~mm}$, respectively.

(a)

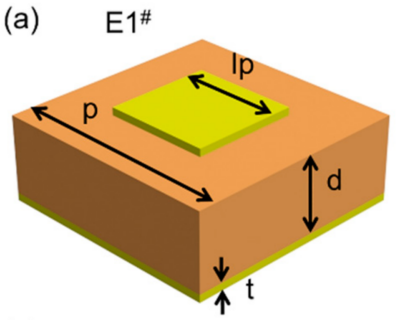

(c)

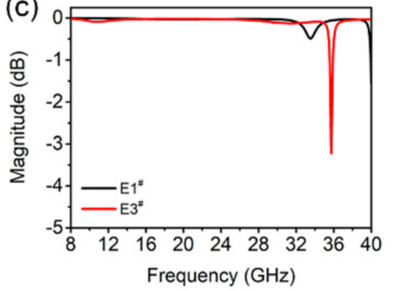

(b)

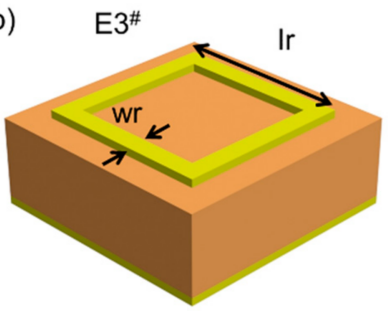

(d)

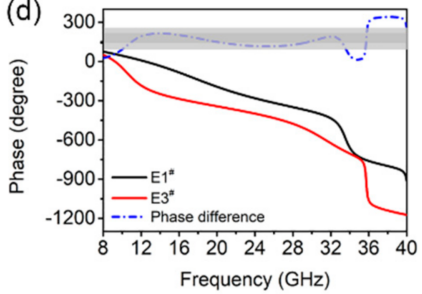

Figure 1. Schematic of the two units of (a) square patch and (b) square ring. The magnitude (c) and phase (d) of reflectivity of the two units.

The reflection coefficient, including amplitude and phase of the electromagnetic wave of the above two units were simulated and presented in Figure 1c,d. From Figure 1c, $\mathrm{E}^{\#}$ and $\mathrm{E} 3^{\#}$ present a narrow band of weak absorption peaks at $33.6 \mathrm{GHz}$ and $35.7 \mathrm{GHz}$, but show strong reflection to the incident electromagnetic wave in other frequency ranges (the reflection coefficient is close to 1 ). Figure $1 \mathrm{~d}$ illustrates the phase difference between $\mathrm{E}^{\#}$ and $\mathrm{E} 3^{\#}$ is $180^{\circ} \pm 60^{\circ}$ ranges within $9-34 \mathrm{GHz}$, which is conducive to RCS reduction. The phase difference between $\mathrm{E} 1^{\#}$ and $\mathrm{E} 3^{\#}$ is $180^{\circ} \pm 37^{\circ}$ in the range of $10.9-20.2 \mathrm{GHz}$ and 29.2-33.1 GHz, which meet the condition of $-10 \mathrm{~dB}$ RCS reduction.

As shown in Figure 2a,b, the structural units with cut-wire patterns arranged at $45^{\circ}$ and $135^{\circ}$ were designed, denoted as $\mathrm{E} 2^{\#}$ and $\mathrm{E} 4^{\#}$, where $l=5 \mathrm{~mm}$ and $w=1 \mathrm{~mm}$. As the two basic units $\mathrm{E}^{\#}$ and $\mathrm{E} 4^{\#}$ are asymmetrical on the $\mathrm{X}$ and $\mathrm{Y}$ axes, their reflected waves produce polarization conversion. The amplitude and phase of the reflected electromagnetic waves are shown in Figure $2 \mathrm{c}, \mathrm{d}$. The co-polarized coefficient and the cross-polarized coefficient is defined as $R_{x x}=\left|E_{x r}\right| /\left|E_{x i}\right|$ and $R_{y x}=\left|E_{y r}\right| /\left|E_{x i}\right|$, respectively. $R_{x x}$ and $R_{y x}$ denote the reflection ratio of $x$-to- $x$ and $x$-to-y polarization conversion, respectively. From Figure $2 \mathrm{c}$ that the $R_{x x}$ (co-polarized coefficient) value of $\mathrm{E} 2^{\#}$ and $E 4^{\#}$ are the same, and the reflectivity is low ranges within $16-29.2 \mathrm{GHz}$. Figure $2 \mathrm{~d}$ shows, however, the value of $R_{y x}$ (cross-polarized coefficient) is close to $0 \mathrm{~dB}$ ranges within $16-29.2 \mathrm{GHz}$, which means an almost total reflection. From the results in Figure $2 \mathrm{c}, \mathrm{d}$, the two units of $\mathrm{E}^{\#}$ and $\mathrm{E}^{\#}$ only exhibit orthogonal polarization reflection in this frequency range. Figure $2 \mathrm{~d}$ also shows that the $R_{y x}$ phase difference of $\mathrm{E}^{\#}$ and $\mathrm{E} 4^{\#}$ is always $180^{\circ}$, which means the same amplitude and opposite phase, so the condition of $-10 \mathrm{~dB}$ RCS reduction is satisfied in the range of $16-29.2 \mathrm{GHz}$. Therefore, the cut-wire patterns arranged at $45^{\circ}$ and $135^{\circ}$ can achieve $180^{\circ}$ phase difference in the maximum frequency range, as shown in Figure $2 \mathrm{~d}$. 
(a) E2

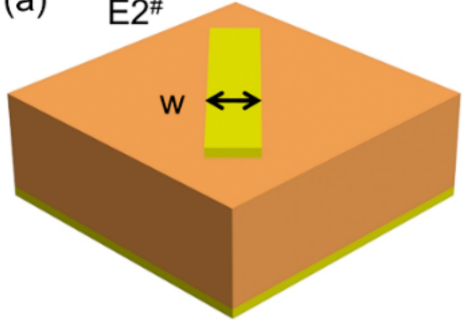

(c)

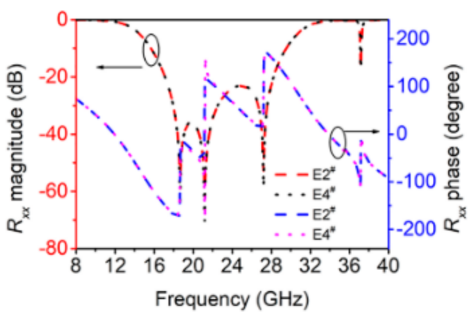

(b)

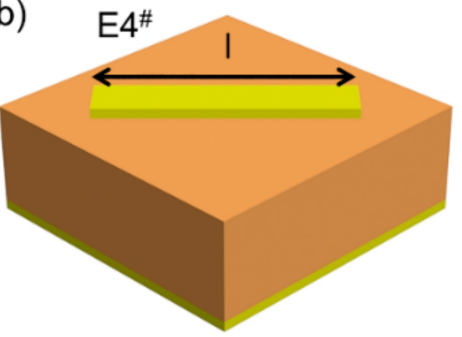

(d)

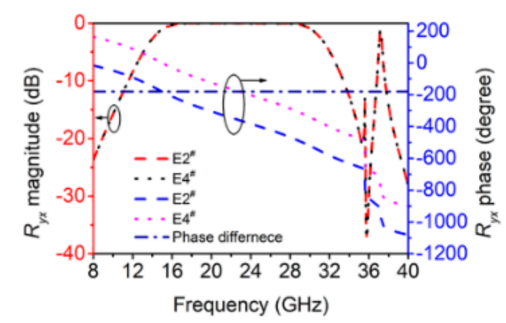

Figure 2. Schematic for the two units of (a) $45^{\circ}$ cut-wire (E2\#) and (b) $135^{\circ}$ cut-wire (E4 $4^{\#)}$. The magnitude and phase of (c) $R_{x x}$ and (d) $R_{y x}$ for $\mathrm{E}^{\#}$ and $\mathrm{E} 4^{\#}$.

\subsection{Binary Metasurface}

As reported by many existing works, a pair of anti-phase unit cells can construct an RCS reduction metasurface that works in their anti-phase band. The above discussed anti-phase pairs, $\mathrm{E}^{\#}, \mathrm{E} 3^{\#}$ and $\mathrm{E}^{\#}, \mathrm{E} 4^{\#}$ are respectively arranged in chessboard-like manner, as shown in Figure 3a. With normal incidence of electromagnetic waves, the RCS reduction of the chessboard-like metasurfaces are simulated as in Figure $3 \mathrm{~b}$. The chessboard metasurface made of $\mathrm{E}^{\#}$ and $\mathrm{E}^{\#}$ shows three strong peaks of RCS reduction at $13 \mathrm{GHz}, 19 \mathrm{GHz}$ and $32 \mathrm{GHz}$, and the maximum reduction reach $-30 \mathrm{~dB}$ at $19 \mathrm{GHz}$. The frequency band with RCS reduction of the metasurface is consistent with the frequency band with $180^{\circ}$ phase difference between $\mathrm{E}{ }^{\#}$ and $\mathrm{E}^{\#}$ units by comparing and analyzing Figures $1 \mathrm{~d}$ and $3 \mathrm{~b}$, which indicate that the RCS reduction is mainly due to the opposite phase of the reflected electromagnetic wave, leading to scattering caused by the interference in the far field. The RCS reduction of the metasurface can reach below $-5 \mathrm{~dB}$ within $9-34 \mathrm{GHz}$, which is almost consistent with the phase difference of $180^{\circ} \pm 60^{\circ}$.

(a)

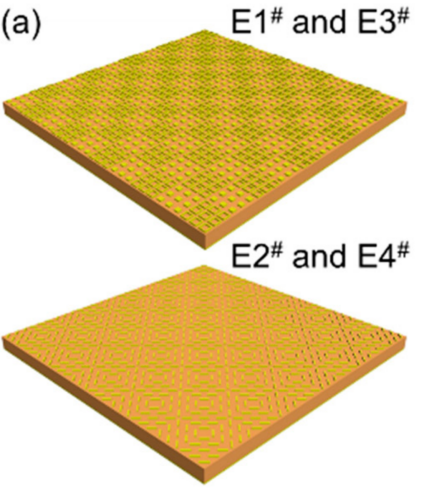

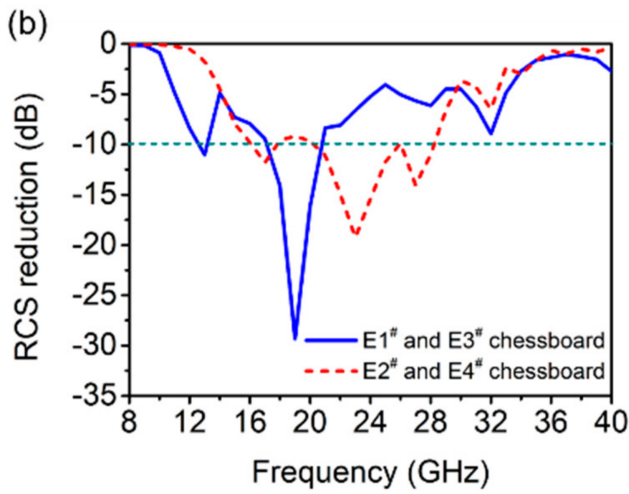

Figure 3. (a) The schematics of $E 1^{\#}, E 3^{\#}$ and $E 2^{\#}, E 4^{\#}$ chessboard metasurface. (b) Simulation result of the RCS reduction of the two chessboard metasurface.

The RCS reduction of chessboard metasurface composed of E2\# and $E 4^{\#}$ can achieve $-10 \mathrm{~dB}$ within $16-29 \mathrm{GHz}$, among which three peaks of the RCS reduction occurs at $17 \mathrm{GHz}$, $23 \mathrm{GHz}$, and $27 \mathrm{GHz}$. Figures $2 \mathrm{~d}$ and $3 \mathrm{~b}$ show that the bandwidth of the RCS reduction is consistent with the frequency of the total reflected cross-polarized electromagnetic wave of $\mathrm{E}^{\#}$ and $\mathrm{E}^{\#}$ units, which further confirms that the RCS reduction of the chessboard 
metasurface composed of $\mathrm{E}^{\#}$ and $\mathrm{E} 4^{\#}$ is owing to the scattering formed by the interference of the cross-polarized reflection waves of the two units ranges within 16-29 GHz.

From the above analysis, the working bandwidth of the RCS reduction of $E 1^{\#}, E 3^{\#}$ and $\mathrm{E} 2^{\#}, \mathrm{E} 4^{\#}$ chessboard metasurface are complementary to each other, which is because that the two pairs of metamaterial units with phase difference of $180^{\circ} \pm 37^{\circ}$ are complementary. On this basis, the two pair of unit cells can be used to construct a quaternionic metasurface to achieve broadened bandwidth of RCS reduction.

\subsection{Quaternionic Metasurface}

According to the design principle of RCS reduction above, the value of RCS reduction of the quaternionic metasurface can be calculated by substituting the reflection amplitude and phase values of $\mathrm{E}^{\#}$ to $\mathrm{E}^{\#}$ into Equation (3), as shown in Figure 4. The theoretical calculation results show that the quaternionic metasurface can achieve less than $-10 \mathrm{~dB}$ RCS reduction in the broadband range of $14-32 \mathrm{GHz}$, and the value of the RCS reduction can reach $-34 \mathrm{~dB}$ and $-23 \mathrm{~dB}$ at $18 \mathrm{GHz}$ and $30 \mathrm{GHz}$, respectively. On the other hand, the weak loss caused by the resonance absorption of the metamaterial unit itself was observed almost $36 \mathrm{GHz}$, which has little effect on the RCS reduction characteristics of the metasurface.

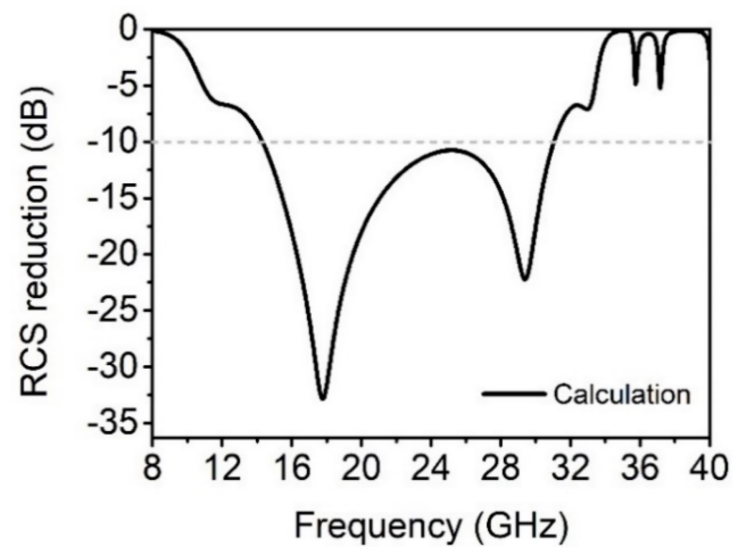

Figure 4. The calculated RCS reduction of the quaternionic metasurface based on Equation (3).

The accuracy of the theoretical design and calculation results of the proposed metasurface was further verified by electromagnetic simulation. The schematic of the quaternionic metasurface, as shown in Figure $5 \mathrm{a}$, where $a=36 \mathrm{~mm}$ and the other parameters are the same as the previous unit structure. The simulation results of RCS reduction of the quaternionic metasurface are illustrated in Figure 5b, which suggest the frequency range of the RCS reduction below $-10 \mathrm{~dB}$ is $14-32 \mathrm{GHz}$. The simulation results are in good accordance with the theoretical calculation, validating the correctness of the design principle. More importantly, the $-10 \mathrm{~dB}$ RCS reduction bandwidth is greatly expanded compared to the performance of the previous binary metasurfaces. The period of a single element in the quaternionic metasurface will affect the bandwidth of RCS reduction. When the period and structure size are reduced, the working frequency band will move to high frequency, and vice versa. If only the area of the full metasurface is enlarged by using different number of periodic elements, its working frequency band will not change. 

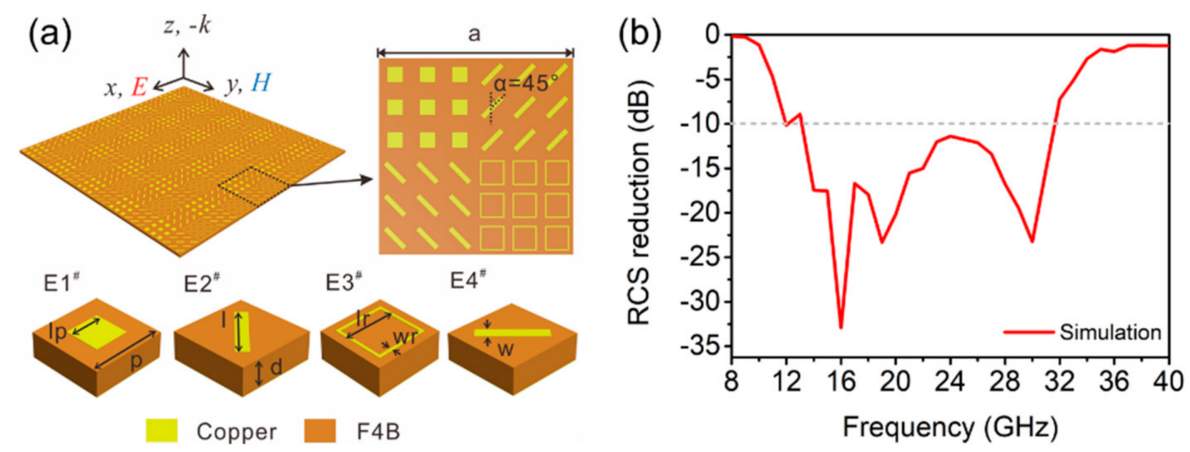

Figure 5. (a) The schematic of the quaternionic metasurface, and (b) simulation result of the RCS reduction.

Figure 6 shows the simulated 3D far-field RCS patterns of the quaternionic metasurface and metal plate at $16 \mathrm{GHz}$ and $30 \mathrm{GHz}$, respectively. At low frequency, the incident wave is mainly scattered into four strong lobes in the $x o z$ and yoz planes and accompanied by some weak lobes. Since most of the electromagnetic energy is scattered to other directions rather than mirror reflection, backward RCS reduction takes effect. From Figure $6 \mathrm{~b}$, d, at high frequency, the perpendicular incident electromagnetic wave is also scattered into many beam lobes, different from the case when the electromagnetic wave incident on the metal plate. Meanwhile, the scattered beam gradually approaches to the center of $\theta=0^{\circ}$ and the scattering phenomenon weakens with the increase of the frequency.
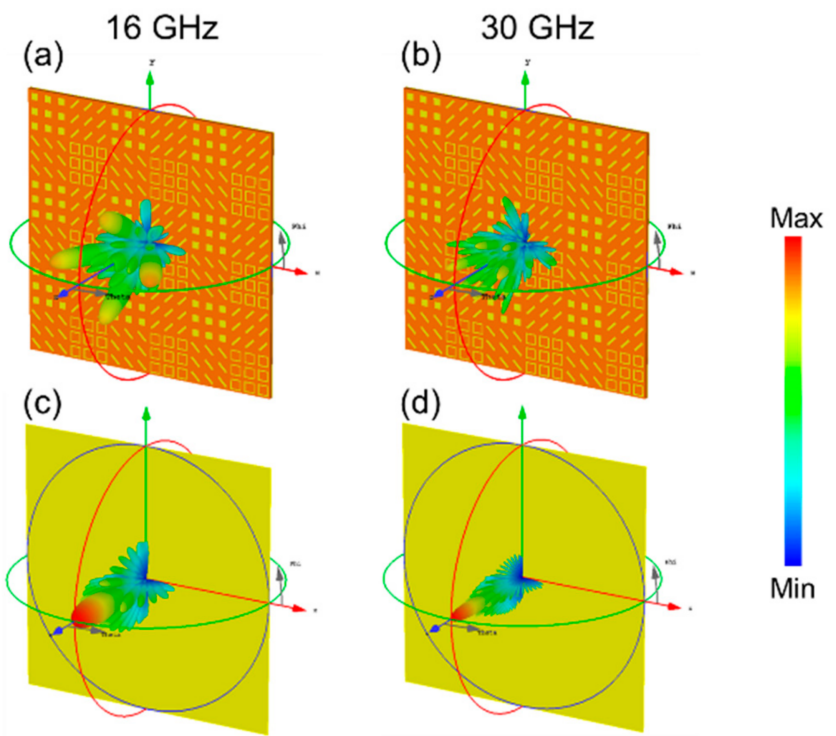

Figure 6. 3D bistatic RCS patterns under normally incident waves for the quaternionic metasurface at (a) $16 \mathrm{GHz}$, (b) $30 \mathrm{GHz}$ and the metal plate at (c) $16 \mathrm{GHz}$, (d) $30 \mathrm{GHz}$.

Figure 7 shows the simulation results of $2 \mathrm{D}$ bistatic RCS of the quaternionic metasurface at $16 \mathrm{GHz}$ and $30 \mathrm{GHz}$, which can be used to quantitatively analyze the effect of RCS reduction of the metasurface. At $16 \mathrm{GHz}$, the strongest reflected beam of the metal plate occurs at $\theta=0^{\circ}$ and its intensity is $7.12 \mathrm{dBsm}$, while the strongest of the metasurface reflected waves are mainly scattered into four beams at $\left(\varphi=0^{\circ} \theta= \pm 30^{\circ}\right)$ and $\left(\varphi=90^{\circ}\right.$ $\theta= \pm 30^{\circ}$ ) and the strength reduced by around $7 \mathrm{dBsm}$. Moreover, the metasurface shows more strong scattering beams at $30 \mathrm{GHz}$, including those at $\varphi=0^{\circ}, 45^{\circ}, 90^{\circ}$ and $135^{\circ}$. In the case of Figure $7 \mathrm{~b}$, the angle of the scattering beam is the same when $\varphi=0^{\circ}$ and $90^{\circ}$, mainly concentrated in $\theta= \pm 15^{\circ}, 35^{\circ}$ and $55^{\circ}$. Nonetheless, when $\varphi=45^{\circ}$ and $135^{\circ}$, the scattered beams increase and disperse, mainly concentrated below $\theta=30^{\circ}$, and two stronger of the side lobes can be observed at $\theta= \pm 22^{\circ}$. At this frequency, the maximum RCS of the 
metasurface is about $8.45 \mathrm{dBsm}$ lower than that of the metal plate. Therefore, it can be confirmed that the scattering effect of the metasurface can effectively reduce the backward RCS of the object.
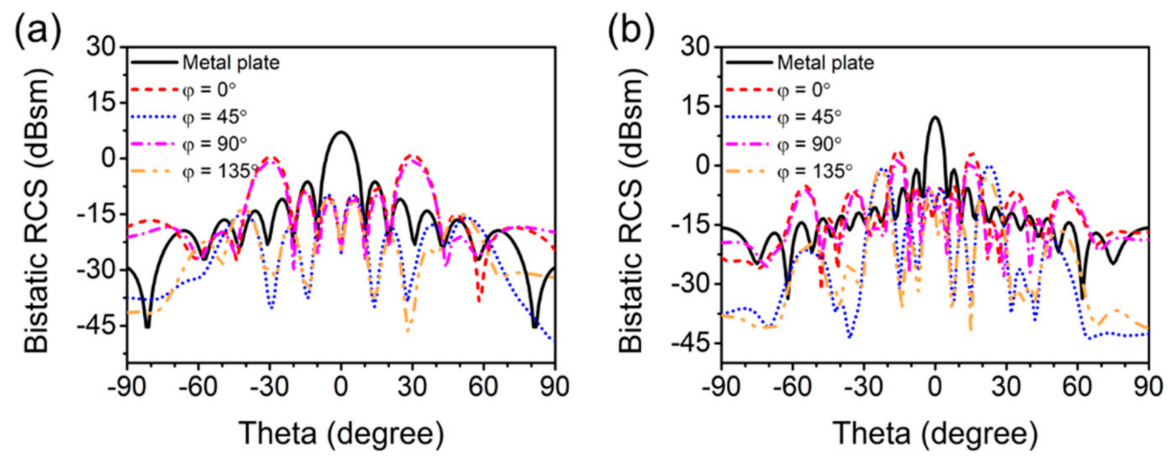

Figure 7. Bistatic RCS versus elevation angle $(\theta)$ for $\varphi=0^{\circ}, 45^{\circ}, 90^{\circ}$ and $135^{\circ}$ cut-planes under normal incidence at (a) $16 \mathrm{GHz}$ and (b) $30 \mathrm{GHz}$.

\subsection{Fabrication and Measurement}

The sample of the quaternionic metasurface with a dimension of $180 \mathrm{~mm} \times 180 \mathrm{~mm}$ $\times 2 \mathrm{~mm}$ were fabricated by traditional PCB technology [32]. In Figure 1a, it is composed of $5 \times 5$ large units arranged periodically. The reflectivity of the fabricated metasurface and metal plate was measured through the NRL-arch method [33], and then was converted into RCS reduction, as shown in Figure $8 b$. The figure indicates that the bandwidth of $-10 \mathrm{~dB}$ RCS reduction is $12.7-33.8 \mathrm{GHz}$, and the relative bandwidth is up to $90 \%$. Small deviations between the measured and the simulated results can be observed, especially at near $30 \mathrm{GHz}$, which is mainly due to the fact that the material parameters of the dielectric plate used are not absolutely consistent with the parameters set by the simulation and the size of the actual sample is slightly different from that of the simulation model. Besides, the simulation curve is drawn by a limited number of points, which is not smooth enough, while the actual measured curve is obtained by hundreds of frequency data. However, the measured results of the metasurface are in good accordance with the simulation results in other frequency ranges, which illuminates that the designed quaternionic metasurface with good RCS reduction effect in the broadband range can be achieved.

(a)

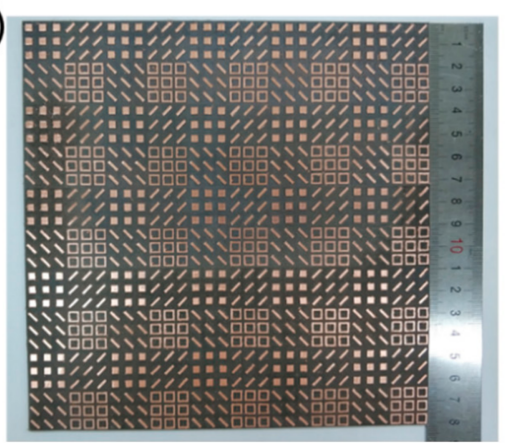

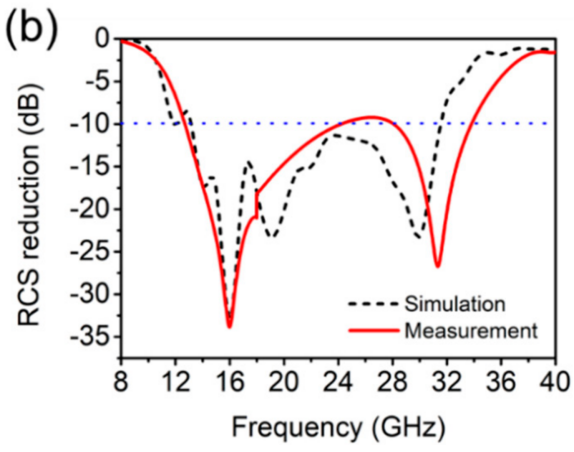

Figure 8. (a) Photograph of the fabricated sample, and its (b) simulated and measured RCS reduction.

\section{Conclusions}

Complementary phase difference wavebands is achieved by a quaternionic metasurface which is composed of two pairs of metasurface unit cells. By combining the two pairs of unit cells with effective phase difference $\left(180 \pm 60^{\circ}\right)$ in different frequency ranges, the bandwidth of radar cross section (RCS) reduction is greatly expanded, while the effective phase difference in the same broadband enhances the RCS reduction. Theoretical calculations, simulations and experiments verified that the less than $-10 \mathrm{~dB}$ RCS reduction 
can be achieved in the broadband of $13-33 \mathrm{GHz}$, and the RCS reduction is mainly achieved by controlling the angular distribution of electromagnetic wave energy rather than absorption. This work provides an effective method for the design of wideband RCS reducer that is important for radar stealth, anechoic chamber and electromagnetic compatibility.

Author Contributions: Conceptualization, Z.Z. and J.G.; methodology, Z.Z. and Y.Z.; software, T.W., Y.Z. and S.C.; validation, Y.Z. and Z.Z.; formal analysis, W.L. and J.G.; investigation, Z.Z. and T.W.; resources, Z.Z.; data curation, Y.Z.; writing-original draft preparation, Z.Z.; writing—review and editing, J.G. and W.L.; visualization, J.G.; supervision, W.L.; project administration, Z.Z.; funding acquisition, W.L. All authors have read and agreed to the published version of the manuscript.

Funding: This work was supported by the National Natural Science Foundation of China (No.51577138), the Fundamental Research Funds for the Central Universities (WUT: 2021IVA116).

Institutional Review Board Statement: Not applicable.

Informed Consent Statement: Not applicable.

Data Availability Statement: Data sharing not applicable.

Conflicts of Interest: The authors declare no conflict of interest.

\section{References}

1. Landy, N.I.; Sajuyigbe, S.; Mock, J.J.; Smith, D.R.; Padilla, W.J. Perfect metamaterial absorber. Phys. Rev. Lett. 2008, 100, 207402. [CrossRef] [PubMed]

2. Luo, J.; Lu, W.; Hang, Z.; Chen, H.; Hou, B.; Lai, Y.; Chan, C.T. Arbitrary control of electromagnetic flux in inhomogeneous anisotropic media with near-zero index. Phys. Rev. Lett. 2014, 112, 073903. [CrossRef] [PubMed]

3. Pfeiffer, C.; Grbic, A. Metamaterial Huygens' surfaces: Tailoring wave fronts with reflectionless sheets. Phys. Rev. Lett. 2013, 110, 197401. [CrossRef] [PubMed]

4. Gao, X.; Han, X.; Cao, W.-P.; Li, H.O.; Ma, H.F.; Cui, T.J. Ultrawideband and high-efficiency linear polarization converter based on double V-shaped metasurface. IEEE Trans. Antennas Propag. 2015, 63, 3522-3530. [CrossRef]

5. Chen, H.-T.; Taylor, A.J.; Yu, N. A review of metasurfaces: Physics and applications. Rep. Prog. Phys. 2016, 79, 076401. [CrossRef]

6. Cai, T.; Wang, G.M.; Xu, H.X.; Tang, S.W.; Li, H.; Liang, J.G.; Zhuang, Y.Q. Bifunctional Pancharatnam-Berry Metasurface with High-Efficiency Helicity-Dependent Transmissions and Reflections. Annalen Phys. 2018, 530, 1700321. [CrossRef]

7. Ishikawa, A.; Tanaka, T. Metamaterial Absorbers for Infrared Detection of Molecular Self-Assembled Monolayers. Sci. Rep. 2015, 5, 12570. [CrossRef]

8. Wang, B.-Y.; Liu, S.-B.; Bian, B.-R.; Mao, Z.-W.; Liu, X.-C.; Ma, B.; Chen, L. A novel ultrathin and broadband microwave metamaterial absorber. J. Appl. Phys. 2014, 116, 094504. [CrossRef]

9. Cheng, Y.; Yang, H. Design, simulation, and measurement of metamaterial absorber. J. Appl. Phys. 2010, 108, 034906. [CrossRef]

10. Yoo, Y.J.; Ju, S.; Park, S.Y.; Kim, Y.J.; Bong, J.; Lim, T.; Kim, K.W.; Rhee, J.Y.; Lee, Y. Metamaterial absorber for electromagnetic waves in periodic water droplets. Sci. Rep. 2015, 5, 14018. [CrossRef]

11. Kowerdziej, R.; Jaroszewicz, L. Tunable dual-band liquid crystal based near-infrared perfect metamaterial absorber with high-loss metal. Liq. Cryst. 2019, 46, 1568-1573. [CrossRef]

12. Bilal, R.; Saeed, M.; Choudhury, P.; Baqir, M.; Kamal, W.; Ali, M.M.; Rahim, A.A. Elliptical metallic rings-shaped fractal metamaterial absorber in the visible regime. Sci. Rep. 2020, 10, 1-12. [CrossRef]

13. Qin, F.; Chen, Z.; Chen, X.; Yi, Z.; Yao, W.; Duan, T.; Wu, P.; Yang, H.; Li, G.; Yi, Y. A tunable triple-band near-infrared metamaterial absorber based on Au nano-cuboids array. Nanomaterials 2020, 10, 207. [CrossRef]

14. Li, Y.; Zhang, J.; Qu, S.; Wang, J.; Chen, H.; Xu, Z.; Zhang, A. Wideband radar cross section reduction using two-dimensional phase gradient metasurfaces. Appl. Phys. Lett. 2014, 104, 221110. [CrossRef]

15. Chen, W.; Balanis, C.A.; Birtcher, C.R. Checkerboard EBG surfaces for wideband radar cross section reduction. IEEE Trans. Antennas Propag. 2015, 63, 2636-2645. [CrossRef]

16. Wen, Q.-Y.; Zhang, H.-W.; Xie, Y.-S.; Yang, Q.-H.; Liu, Y.-L. Dual band terahertz metamaterial absorber: Design, fabrication, and characterization. Appl. Phys. Lett. 2009, 95, 241111. [CrossRef]

17. Sellier, A.; Teperik, T.V.; de Lustrac, A. Resonant circuit model for efficient metamaterial absorber. Opt. Express 2013, 21 (Suppl. 6), A997-A1006. [CrossRef]

18. Shen, X.; Cui, T.J.; Zhao, J.; Ma, H.F.; Jiang, W.X.; Li, H. Polarization-independent wide-angle triple-band metamaterial absorber. Opt. Express 2011, 19, 9401-9407. [CrossRef]

19. Ding, F.; Cui, Y.; Ge, X.; Jin, Y.; He, S. Ultra-broadband microwave metamaterial absorber. Appl. Phys. Lett. 2012, 100, 103506. [CrossRef]

20. Yin, X.; Long, C.; Li, J.; Zhu, H.; Chen, L.; Guan, J.; Li, X. Ultra-wideband microwave absorber by connecting multiple absorption bands of two different-sized hyperbolic metamaterial waveguide arrays. Sci. Rep. 2015, 5, 1-8. [CrossRef] 
21. Long, C.; Yin, S.; Wang, W.; Li, W.; Zhu, J.; Guan, J. Broadening the absorption bandwidth of metamaterial absorbers by transverse magnetic harmonics of 210 mode. Sci. Rep. 2016, 6, 21431. [CrossRef] [PubMed]

22. Li, W.; Wu, T.; Wang, W.; Zhai, P.; Guan, J. Broadband patterned magnetic microwave absorber. J. Appl. Phys. 2014, 116, 044110. [CrossRef]

23. Chen, K.; Cui, L.; Feng, Y.; Zhao, J.; Jiang, T.; Zhu, B. Coding metasurface for broadband microwave scattering reduction with optical transparency. Opt. Express 2017, 25, 5571-5579. [CrossRef] [PubMed]

24. Huang, C.; Zhang, C.; Yang, J.; Sun, B.; Zhao, B.; Luo, X. Reconfigurable metasurface for multifunctional control of electromagnetic waves. Adv. Opt. Mater. 2017, 5, 1700485. [CrossRef]

25. Zhao, J.; Sima, B.; Jia, N.; Wang, C.; Zhu, B.; Jiang, T.; Feng, Y. Achieving flexible low-scattering metasurface based on randomly distribution of meta-elements. Opt. Express 2016, 24, 27849-27857. [CrossRef] [PubMed]

26. Al-Nuaimi, M.K.T.; Hong, W.; He, Y. Backscattered EM-wave manipulation using low cost 1-bit reflective surface at W-band. J. Phys. D Appl. Phys. 2018, 51, 145105. [CrossRef]

27. Zhao, J.; Cheng, Q.; Wang, X.K.; Yuan, M.J.; Zhou, X.; Fu, X.J.; Qi, M.Q.; Liu, S.; Chen, H.B.; Zhang, Y. Controlling the bandwidth of terahertz low-scattering metasurfaces. Adv. Opt. Mater. 2016, 4, 1773-1779. [CrossRef]

28. Moccia, M.; Liu, S.; Wu, R.Y.; Castaldi, G.; Andreone, A.; Cui, T.J.; Galdi, V. Coding metasurfaces for diffuse scattering: Scaling laws, bounds, and suboptimal design. Adv. Opt. Mater. 2017, 5, 1700455. [CrossRef]

29. Paquay, M.; Iriarte, J.-C.; Ederra, I.; Gonzalo, R.; de Maagt, P. Thin AMC structure for radar cross-section reduction. IEEE Trans. Antennas Propag. 2007, 55, 3630-3638. [CrossRef]

30. Zhao, J.; Zhang, C.; Cheng, Q.; Yang, J.; Cui, T.J. An optically transparent metasurface for broadband microwave antireflection. Appl. Phys. Lett. 2018, 112, 073504. [CrossRef]

31. Cui, T.J.; Qi, M.Q.; Wan, X.; Zhao, J.; Cheng, Q. Coding metamaterials, digital metamaterials and programmable metamaterials. Light Sci. Appl. 2014, 3, e218. [CrossRef]

32. Mattelin, A. Method for Manufacturing Printed Circuit. Boards.U.S. Patent No. 4,943,346, 24 July 1990.

33. Knott, E.F.; Schaeffer, J.F.; Tulley, M.T. Radar Cross Section; SciTech Publishing: Raleigh, NC, USA, 2004. 